\title{
A comparison of sensors for underground void mapping by unmanned aerial vehicles
}

\author{
F Azhari Commonwealth Scientific and Industrial Research Organisation, Australia \\ S Kiely Commonwealth Scientific and Industrial Research Organisation, Australia \\ C Sennersten Commonwealth Scientific and Industrial Research Organisation, Australia \\ C Lindley Commonwealth Scientific and Industrial Research Organisation, Australia \\ M Matuszak University of Tasmania, Australia
}

S Hogwood University of Tasmania, Australia

\begin{abstract}
Unmanned aerial vehicles (UAVs) are being employed in a rapidly increasing number of applications in mining, including the emerging area of mapping underground void spaces such as stopes, which are otherwise inaccessible to humans, automated ground vehicles and survey technologies. Void mapping can provide both visual rock surface and $3 D$ structural information about stopes, supporting more effective planning of ongoing blast designs. Underground stope mapping by UAVs, however, involves overcoming a number of engineering challenges to allow flights beyond operator line-of-sight where there is no global positioning system (GPS), natural or artificial light, or existing communications infrastructure.
\end{abstract}

This paper describes the construction of a UAV sensor suite that uses sound navigation and ranging (SONAR) data to create a rough 3D model of the underground UAV operational environment in real time to provide operators with high situational awareness for beyond line-of-sight operations. The system also provides a backup when dust obscures visual sensors to provide situation awareness and a coarser, but still informative, 3D model of the underground space.

Typically, light detection and ranging (LIDAR) systems have superseded SONAR sensors for similar applications. LIDAR is much more accurate than SONAR, but has several disadvantages. SONAR sensor data is sparse, and therefore much easier to process in real time on-board the UAV than LIDAR. The SONAR sensor hardware is also lighter than current LIDAR systems, which is of importance regarding the constrained payload capacity of UAVs. However, the most important factor that makes SONAR stand out in this application is its ability to operate in dusty or smoke-filled environments.

The UAV system was tested both above and below-ground using a predefined path with check point locations for the UAV to follow. Due to the lack of GPS, survey points in combination with photogrammetry allowed the UAV's location to be estimated. This allowed the system to be tested to determine how accurate the SONAR data is in comparison with $3 D$ modelling via photogrammetry of images from a separate digital single-lens reflex camera.

Comparing the shape of void surfaces determined by photogrammetry with that determined by SONAR provides quantifiable accuracy when the photogrammetry models are used as ground truth data. Above-ground and underground pilot studies have determined that SONAR sensors provide acceptable accuracy compared with modelling via photogrammetry, sufficient to provide effective situational awareness for human operation of the UAV beyond line-of-sight.

Keywords: SONAR, UAV, 3D mapping, underground, global navigation satellite system 


\section{Introduction}

There are many advantages that unmanned aerial vehicles (UAVs) may bring in underground situations such as underground mines. Applications such as 3D mapping and underground exploration by UAVs hold a lot of interest in the mining community. UAVs have the ability to enter spaces that are inaccessible to people or other equipment, and are suited to explore vertical structures, such as stopes, ore passes, shafts and raises. A primary example of potential routine use of UAVs underground is for stope mapping. Stopes are inaccessible for human entry due to their lack of roof protection and potential instability. However, assessment of blasted stope shape and comparison of that shape with an intended stope design can greatly benefit ongoing drill and blast design, with a view to minimising under and overbreakage. Underbreakage results in loss of ore and therefore, reduced revenue, while overbreakage results in ore dilution (Hustrulid \& Bullock 2001, Table 70.3), presenting a $93.3 \%$ decrease in cash flow for $35 \%$ dilution, which decreases to a $19 \%$ decrease in cash flow for $10 \%$ dilution. This represents a massive productivity improvement.

Currently, stope mapping is routinely done from the base of a stope using a laser scanner mounted on a boom. This has the limitations of decreasing accuracy with distance from the scanner, and shadowing effects due to convex shapes on the stope walls. UAVs have the advantage of being able to fly up into a stope, avoiding shadowing, as well as being able to provide visual data for assessment of structural features and mineralogical characteristics. In the mining industry, 3D mapping of unknown spaces beyond line-ofsight can also ensure the safety of miners and expensive mining equipment. There are many advantages in using UAV technology for mapping newly mined or abandoned spaces, since these areas are hazardous for humans, may have unstable roofs and walls, contain unknown terrain unsuitable for ground vehicles, may be flooded, have an unknown depth, or contain harmful chemicals. UAV 3D mapping technology will not only allow the exploration and mapping of unknown spaces but also allow the maps of known areas to be updated regularly and efficiently to determine changes in the structure of voids, due, for example, to rock falls or convergence. This results in better planning of the mine, and helps with the design of engineering solutions for stability and acid water runoff for example.

However, lack of a positioning system such as global navigation satellite systems (GNSS), or a communications infrastructure for operating beyond line-of-sight (BLOS), or the potential presence of dust in the atmosphere can drastically inhibit the practicality of UAVs for these applications. The study reported in this paper aimed to determine the reliability of sound navigation and ranging (SONAR) systems on UAVs in an underground context as a possible source of positioning information, inspired by SONAR-based 3D mapping studies in other mobile robot contexts (Elfes 1990).

There are underground mines equipped with GNSS analogues, such as the Kankberg mine in Sweden, operated by Boliden and developed by Ericsson, that use triangulation to very low frequency (VLF) radio sources to detect positions with an accuracy of up to $3 \%$ and over distances up to $2 \mathrm{~km}$ (InfoMine 2008). However, reliable local methods are needed, especially if a vehicle needs to process the data in real time for localisation and mapping in confined spaces, and to provide BLOS operators with situation awareness in real time; triangulation in real time cannot, in any case, provide the required void surface data.

Photogrammetry can be used to reconstruct a 3D model of a mine void. This method needs suitable lighting, cannot work if airborne dust obscures visual features of surfaces, and is challenging to process fast enough to support real-time operation. Hence, there are restrictions in the use of photogrammetric techniques to obtain positional data, for 3D mapping, and to avoid crashing a UAV into obstacles. SONAR sensors do not need light, can operate through dust, and generate sufficiently sparse data for straightforward real-time processing. Hence, SONAR sensors, together with inertial measurement data, have the potential to operate under these circumstances. However, if SONAR is used, it is necessary to mathematically characterise its accuracy and limitations. For this, photogrammetrically derived 3D models from a well-lit mine void, with negligible dust and processed offline, provide a reasonable reference and ground truth for comparison and quantification of SONAR system performance.

The UAV used in the study uses WiFi for communication with a 150 Mbps module. This is sufficient to stream compressed video at an $18 \mathrm{~Hz}$ frame rate and telemetry data over a radio link. 
The paper presents the UAV and its sensors in Section 2, followed by the experiment design in Section 3. Thereafter, the two experiments are presented in Sections 4 and 5, respectively. The results of the experiments can be found in Section 6 and are discussed in Section 7.

\section{UAV set-up and sensors}

The following subsections describe the set-up of the UAV, including all of its sensors.

\subsection{UAV with mounted sensors}

The UAV used for the experiment was a custom built quadrotor in an ' $X$ ' configuration, based upon the DJI F450 frame. The X-configuration had four arms with motors mounted $90^{\circ}$ apart, with a $45^{\circ}$ vertical rotation of each pair of arms from the front axis. This left the front of the UAV open for the camera and other sensors, allowing a clear line-of-sight with no platform obstructions.

The UAV carried a global shutter monocular camera (the IDS UI-1222LE) that was used for robust visual inertial odometry (ROVIO) (Bloesch et al. 2015). Since there is minimal available light in a typical underground environment, the UAV carried two 32 W LED lights that could provide sufficient light for visual data collection at up to $6 \mathrm{~m}$, with an absolute maximum of approximately $8 \mathrm{~m}$. This range required a very sensitive camera with a large sensor. The global shutter camera was chosen to minimise the distortion of the image, since the UAV is a dynamic system and in constant motion.

Since the UAV was designed to explore inaccessible areas, it required reliable position information. A number of existing visual simultaneous localisation and mapping (SLAM) (Thrun et al. 2005) solutions were evaluated, such as parallel tracking and mapping (PTAM) (Klein \& Murray 2007), large-scale direct monocular SLAM (LSD-SLAM) (Engel et al. 2014) and semi-direct visual odometry (SVO) (Forster et al. 2014). Most of them suffer from poor performance with only rotational motion and no translation. The ROVIO team solved this problem by fusing visual SLAM data with inertial measurement unit (IMU) data, which provides much better performance. The ROVIO software ran on a Linux mobile computing platform ODROID XU3, and was capable of processing 18 frames per second (fps) of video data.

The flight controller used in the UAV was Pixhawk with a PX4 firmware stack. This provided attitude (i.e. orientation with respect to the direction of travel or an external coordinate reference frame) and position control.

For this experiment, the UAV was also equipped with six SONAR sensors. The SONARs were placed on the ends of each quadrotor arm as well as above and below (Figure 1). This configuration was pre-designed and optimised for wall-following flights, since the UAV was in a former set-up designed to maintain a $45^{\circ}$ yaw angle away from the wall, allowing the front camera to keep the wall and any obstacles in field-of-view. This configuration allowed one SONAR sensor to face the wall directly, thus optimising the distance measurement accuracy. The horizontal SONAR sensors are close to motors and propellers. To protect them from vibrations and propeller noise the sensors were housed inside 3D printed enclosures with vibration isolation, with openings only in the sensing direction. The horizontal SONARs were $47 \mathrm{~cm}$ from the UAV's centre point, the top SONAR $4 \mathrm{~cm}$ above and the bottom $10 \mathrm{~cm}$ below.

SONAR measurements are relatively slow compared to the IMU, since the sensor takes time to average samples until a reliable result is obtained. Reflections and reverberations within the operating space require a delay before subsequent measurements can be taken. Thus, measurements were separated by a gap of $80 \mathrm{~ms}$ and had to be conducted one at a time so that reflections from the previous SONAR reading did not affect the current reading. This limited the SONAR data rate to approximately $2 \mathrm{~Hz}$. In comparison, the IMU streamed the data at a rate of $250 \mathrm{~Hz}$. Assuming the vehicle travels at $1 \mathrm{~m} / \mathrm{s}$, it will therefore travel $0.5 \mathrm{~m}$ between SONAR measurements and $4 \mathrm{~mm}$ between IMU measurements. 


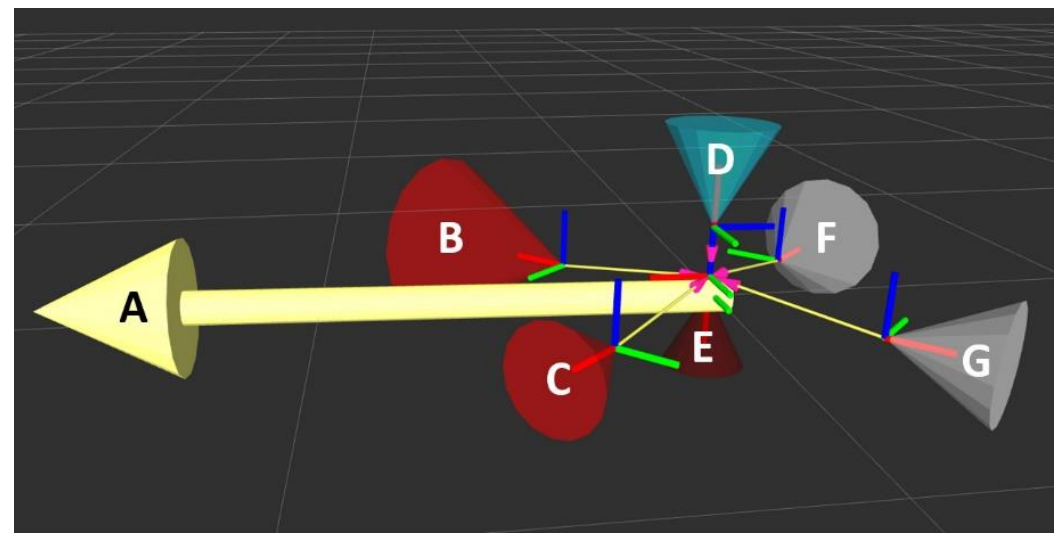

Figure 1 Placement of SONAR sensors. The yellow arrow (A) represents the forward direction; red (B, C) and grey $(F, G)$ colours show the SONAR sensors around the yaw plane separated by $90^{\circ}$ and rotated around the vertical (Z) axis by $45^{\circ}$; the blue (D) and dark red (E) represent the top and bottom SONARs on the $Z$ axis

\subsection{IMU}

The IMU data was collected from the Pixhawk flight controller with a sampling rate of $250 \mathrm{~Hz}$, and the data was recorded by the on-board Odroid computer. The Pixhawk uses an MPU6000 microelectromechanical systems (MEMS) gyroscope and accelerometer that are integrated onto a single chip. It provided the following measurements:

- Three-axis linear acceleration $\left(\mathrm{m} / \mathrm{s}^{2}\right)$.

- Three-axis angular velocity (rad/s).

\subsection{SONAR}

There were six SONAR sensors on-board the UAV, as described previously. The side and bottom sensors were of model MB1242 (MaxBotix Inc. 2012a) and the top one was MB7040 (MaxBotix Inc. 2012b), both designed and manufactured by MaxBotix Inc. These SONAR devices measure the time of travel of a reflected sound impulse from an obstacle. There may be multiple echoes in the return signal, but only the first is taken into consideration.

The MB1242 model has a wider detection beam, but its electronic components are exposed. The MB7040 has a very narrow beam and its design protects it from water dripping from above. The detection distance of the SONAR depends on the size of object reflecting the SONAR pulse, and large objects can be detected at a greater distance than small objects. This is linked to the amplitude of the reflected signal. The MB1242 and MB7040 devices have a maximum detection range of 6.4 and $7.5 \mathrm{~m}$, respectively, and human-size objects are detectable to about $2 \mathrm{~m}$ for MB1242 and $3 \mathrm{~m}$ for MB7040. The distance measurements taken by the SONARs were recorded by the on-board Odroid computer.

\subsection{On-board camera}

The on-board camera was a monochromatic charge-coupled device (CCD) with a global shutter that ran at a $752 \times 480$ resolution at $18 \mathrm{fps}$, and had a lens with a $120^{\circ}$ field-of-view. The camera had a $7 \mu \mathrm{m}$ (micrometre) pixel size and was well-suited for operation in a low light environment. The camera can exceed more than $60 \mathrm{fps}(60 \mathrm{~Hz})$, but the maximum frame rate was limited by the computational power of the Odroid computer to $18 \mathrm{fps}(18 \mathrm{~Hz})$. The IMU timestamp and camera frame timestamp were synchronised to make sure they both ran against the same clock source.

Raw images were used for visual odometry and recording into a bag file. Compressed images were streamed to a ground station Linux computer. 


\subsection{Light source}

Two 32 W XHP-70 LEDs (Cree, Inc. 2017) provided the light source for the camera, which generated over 4,000 lumens each. The high power consumption of these additional two light sources, as well as the extra weight of a cooling system to cool them, significantly limited the flight time to approximately 8 min, which is $50 \%$ of the UAV's maximum flight time without lights.

\section{Experiment design}

Two experiments were conducted $750 \mathrm{~m}$ below the surface at the $17^{\text {th }}$ underground level in the MMG Rosebery mine, Tasmania (13 December 2016).

A pre-mark-up session was first conducted with fiducial/contrast markers in the underground space before the actual trial started (see Figure 2). This was to collect images for photogrammetry supporting the SONAR baseline using a digital single-lens reflex camera (DSLR) that surveyed the underground void spaces where the experiments took place.

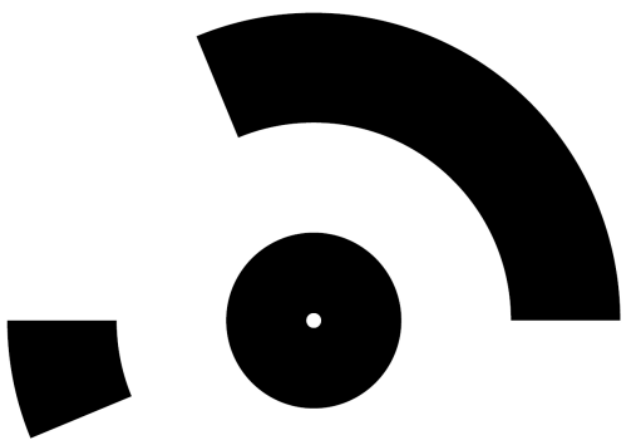

Figure 2 The fiducial/contrast markers used in the underground space

Every marker carried a unique pattern so that a post-processed procedure could identify their location. Both experiments began by measuring out a straight, $15 \mathrm{~m}$ course along the floor of the mine drive with 'start', '1st', '2nd' and 'end' cones. These cones were aligned with the course and positioned $5 \mathrm{~m}$ apart to be used as flight waypoints (Figure 3). In order to perform a comparison between the datasets, a baseline was necessary as a reference.

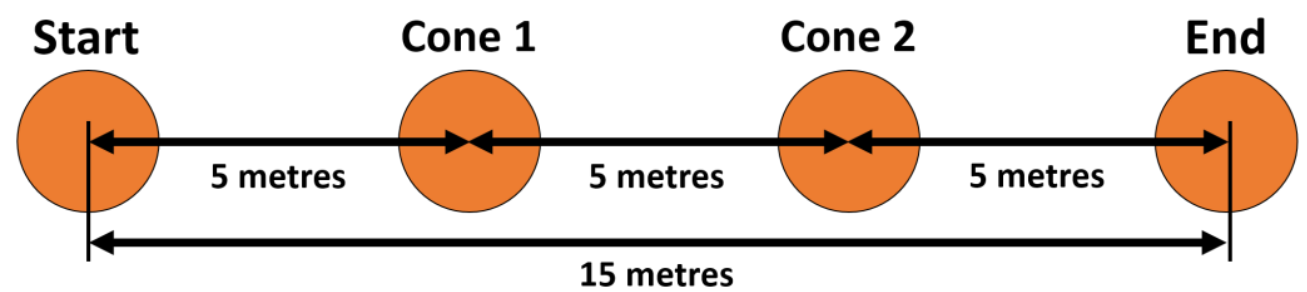

Figure 3 Baseline: a carried flight path and an intended flown flight path

A laser was positioned at the start of the course pointing towards the end cone. This laser was situated at a height of $1 \mathrm{~m}$ so that it could be used as a target reference for the UAV, allowing it to be kept at a consistent height during the experiment. Throughout both experiments, the same procedure was followed, with the exception that the first experiment involved carrying the UAV, whilst during the second experiment, the UAV was flown. During both experiments the laser was used as a reference to allow the UAV to be kept along a consistent and straight path while being carried or flown along the $15 \mathrm{~m}$ course. Two similar path modes were tested in each experiment; the first, referred to as the 'wallaby', consisted of a series of short UAV 'hops'. Meanwhile the other, referred to as the 'kangaroo', consisted of a singled ascent followed by a linear traversal of the path and the landing (one long hop). 


\subsection{Procedure and logging for the 'wallabies'}

The UAV was first positioned on the ground at the start location of the $15 \mathrm{~m}$ path, without any rotor blades mounted. The operator's Linux computer with communication and radio contact were first checked to ensure full contact via RViz (Open Source Robotics Foundation, Inc. 2017), a visualisation tool in Robot Operating System (ROS). The radio controller (RC) was turned on, and shortly after was armed/started. With the UAV on the ground, RC logging started, marking positions/locations, each record constituting a mark. The UAV was then lifted or flown up to the height of the laser, where another mark was made using the RC.

Next, the UAV was carried/flown forward towards cone one, taking care to keep the drone inline with the laser pointer. Once the UAV was positioned above cone one while still inline with the laser pointer, another mark was made using the controller. The UAV was then set on the ground next to cone one, and another mark was made. The drone was then picked/flown back up so it was again inline with the laser and above cone one, and an additional mark was made. This procedure was repeated for all cones through to the end point, where, after the final mark, the RC was then disarmed and the logging procedure was over.

\subsection{Procedure and logging for the 'kangaroo'}

Using the same path as previously, and positioning the UAV at the start on the ground, a mark was recorded, and the UAV was lifted/flown up and aligned with the laser $1 \mathrm{~m}$ above the floor and another mark recorded. This time the height was maintained, but motion was paused above each cone while a mark was made. This was done while the UAV was still aligned with the laser rather than touching the floor. The same procedure was carried out along the whole path, and finally, at the end, it was set down where the final mark was made. The difference with this second procedure was the exclusion of the actions of placing the UAV on the floor at each intermediate cone location (cone one and cone two). Instead, the UAV was taken inline with the laser throughout the entire procedure, and only a single mark was made while the drone was above each cone, rather than three marks at each cone, as in the previous wallaby procedure.

\section{$4 \quad$ Experiment 1}

The first round of data capture underground was located at Level 17 at a pump station at $850 \mathrm{~m}$ depth in the Rosebery mine on the west coast of Tasmania, Australia.

The distance was measured, the cones located, and the laser placed at the start point with an end plate at the end point for ensuring the correct height through the $15 \mathrm{~m}$ path. After this, contrast markers were placed on the wall and the floor, located by the cones. These markers were manually photographed and captured via the UAV's front camera. The images taken from the manual camera used a flash, and the UAVcaptured images needed additional light, so a light source was mounted on the UAV to support this.

Starting the computer log, the RC and the UAV first, the locations of the UAV and its relation to space/volume were ready for capture. The UAV flew and was carried along the predefined path several times, as described in the experiment section, with both the 'wallaby' and 'kangaroo' methods.

The markers on the wall and the floor were later surveyed by an MMG surveyor, which provided correct local and global locations in the mine.

\subsection{Surveying data with location information}

The randomly placed markers were surveyed by one of MMG's surveyors. This gave the local and global position of each marker, which were subsequently referred to as 'survey point/s'. Photos taken using DSLR were stitched together using Agisoft PhotoScan ${ }^{\text {TM }}$ (Agisoft LLC 2017) photogrammetry software to create a $3 \mathrm{D}$ model of the void where the experiment was conducted. Aligning the markers detected on the 3D model with the surveyed points allowed the size, orientation and translation of the 3D model to be corrected. The resulting 3D model of the void served as a baseline for evaluating the relative accuracy of the SONARs mounted on the UAV. 


\subsection{Logging IMU}

IMU logging resulted in 10 rounds of data, but only the two best rounds of the experiment are exemplified (Figures 4 and 5). These were the initial measurements from the Rosebery mine at Level 17 on 13 December 2016.

Only a single test run of the experiment provided reasonable positional data from the IMU (Figure 4).

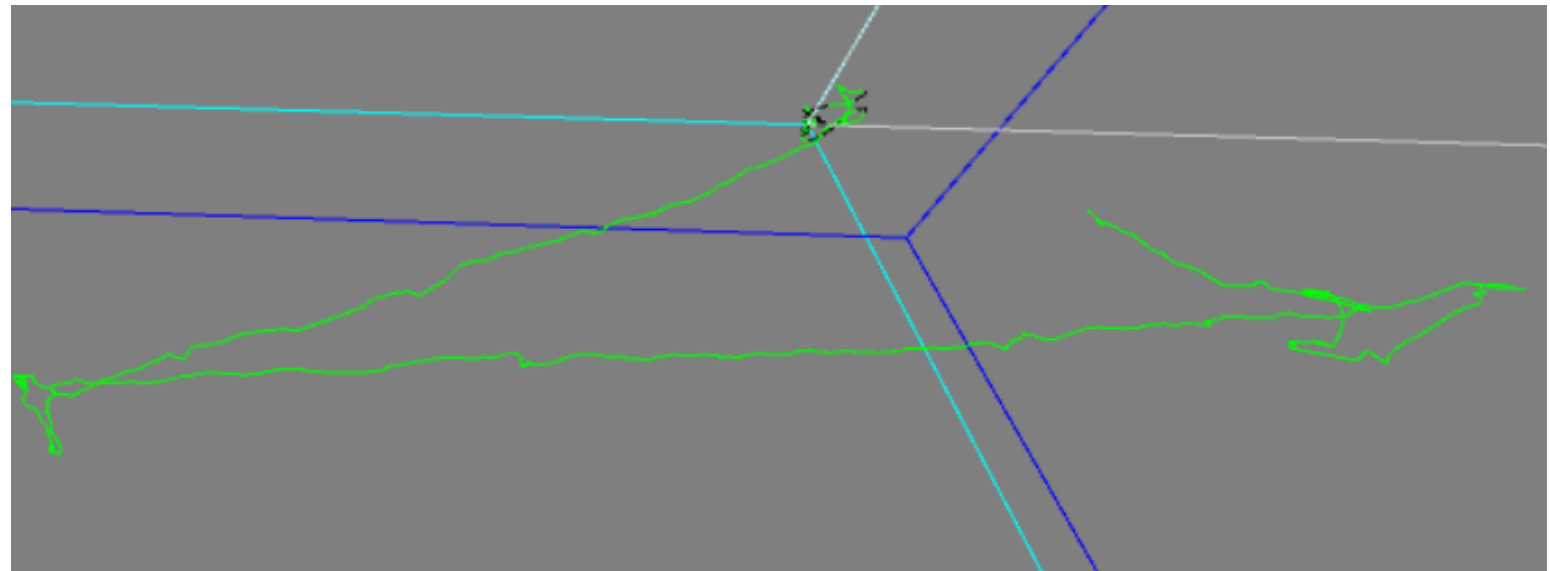

\section{Figure 4 Representation of UAV trajectory}

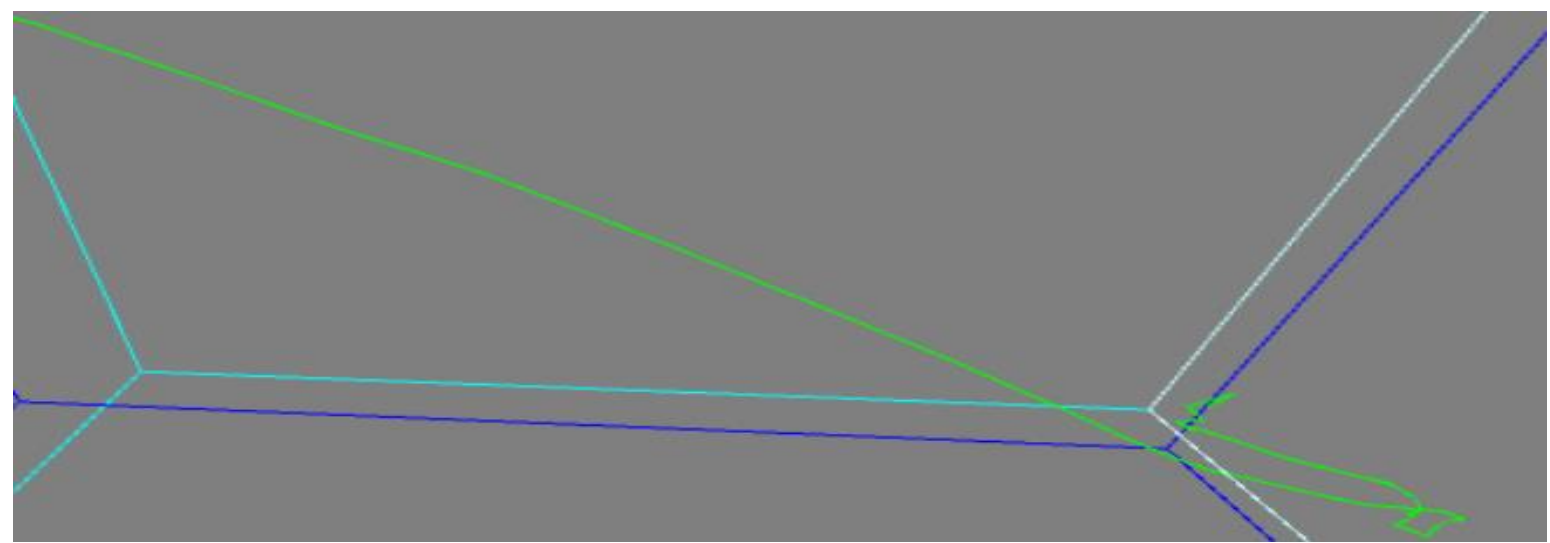

\section{Figure 5 Erroneous trajectory data due to insufficient light}

In the remaining test runs, the IMU data was unreasonable due to large amounts of drift that occurred when the UAV was operating with insufficient light. The drift can be seen in the data as the path incorrectly showing the UAV moving rapidly in a random direction for significantly large distances. This drift can be seen in Figure 5, where the UAV's location appears to travel towards the upper-left portion of the figure. In this example, the UAV's path continued well past the borders of the figure, resulting in a drift in the order of kilometres away from its actual location.

\subsection{Logging SONAR}

The SONAR data was collected in the form of a distance (in metres) between the sensor and the object detected at each sample time. Thus, in order for this data to have any significance, it had to first be aligned with the IMU data. Figure 6 shows the visualisation after the data are aligned with the 3D model of the void. 


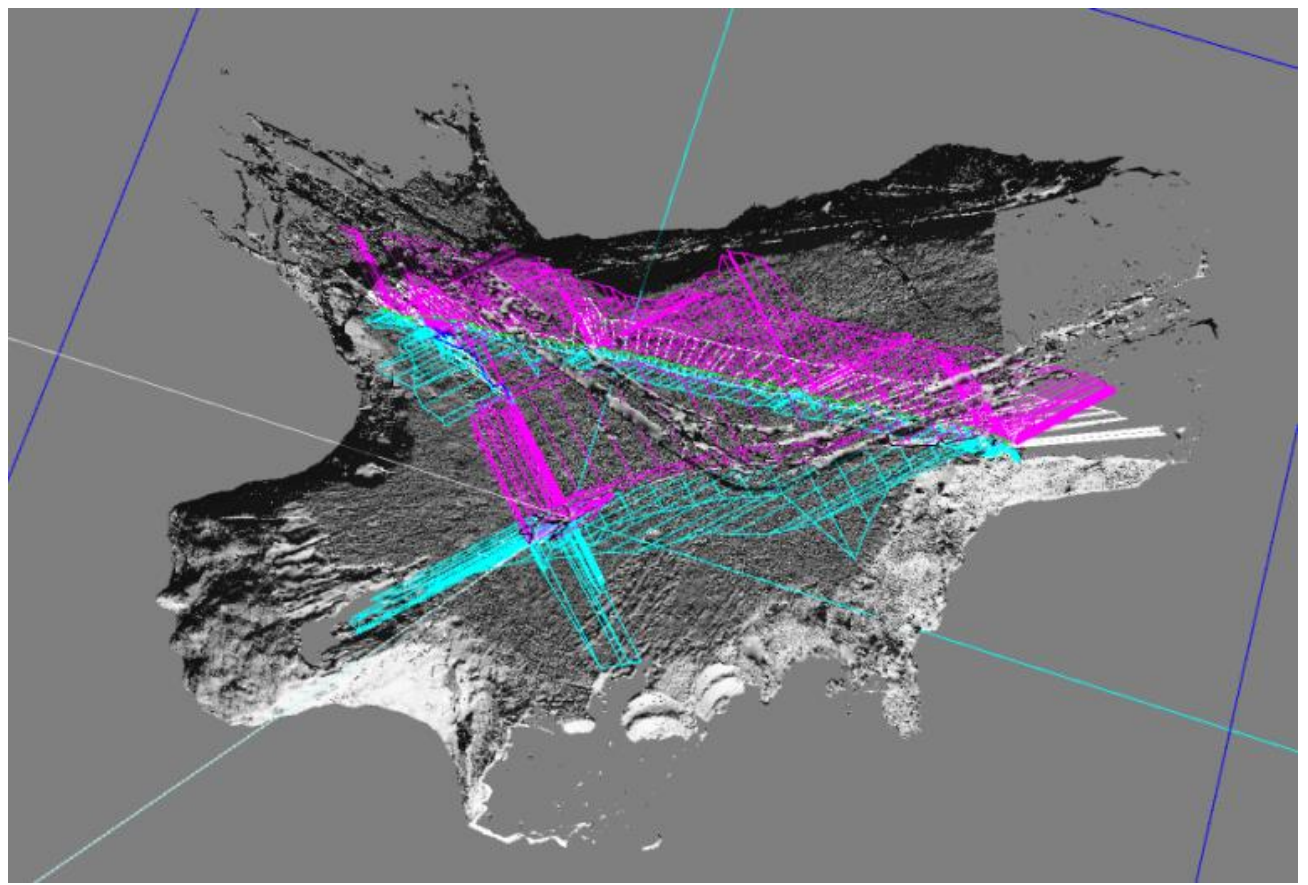

Figure 6 Top view of distances measured by SONAR sensors; cyan for the front and rear-left sensors, and magenta for the front and rear-right sensors

\subsection{Aligning IMU data and SONAR data}

The data obtained from the UAV that was used to calculate the location of the obstacles that the SONAR was sensing consisted of the IMU's local position, the IMU's orientation in the form of a quaternion, the distance each SONAR sensor is from the IMU, the orientation of each SONAR relative to the IMU in the form of a quaternion, and lastly, the distance between the SONAR sensor and the obstacle it is detecting. Since the IMU's location could be aligned with the photogrammetry mesh, which in-turn was already aligned with the survey points, the global position of any obstacle detected by the SONAR sensors on the UAV could be determined using relative vector and quaternion arithmetic, as shown in Figure 6.

\subsection{Constraints}

In relation to the positioning algorithm, the algorithm failed to produce a reliable path compared to the path from the UAV being carried due to the lighting conditions, since the algorithm fuses both IMU data and images from the on-board camera (Section 2.1).

The lighting conditions in Experiment 1 were poor due to electrical noise coming from the power supply. The noise caused a short supply of power to the camera which reduced the frame rate of the camera to as low as $1 \mathrm{fps}$. This was fixed in Experiment 2 by adding an electromagnetic interference filter to the power supply.

\section{$5 \quad$ Experiment 2}

Experiment 2 was conducted upon return to Level 17 one month later, but this time at another location in the mine. It was understood, after analysing the results from Experiment 1, that the range of these particular SONAR sensors was limited to $3 \mathrm{~m}$, so a narrower void/drive to capture full coverage between the SONAR and the IMU-based 3D model was needed.

The distance was measured, the cones located, and the laser placed at the start point with an end plate at the end for correct height through the $20 \mathrm{~m}$ path. Then markers were placed as in the former experiment. The markers were manually photographed, and were also captured via the UAV's front camera. 
The computer log, RC and the UAV were started prior to capturing the locations of the UAV and its relation to space/volume. The UAV flew and was carried along the predefined path several times, as described in the experiment section with both the 'wallaby' and 'kangaroo' procedures.

The markers on the wall and the floor were later surveyed by an MMG surveyor, which provided correct local and global locations in the mine.

\subsection{Surveying data with location information}

Similar to Experiment 1, see Subsection 4.1.

\subsection{Logging IMU}

Similar to Experiment 1, a subset of the test runs giving usable IMU data, for the same reasons listed in Section 4.2.

\subsection{Logging SONAR}

Similar to Experiment 1, see Subsection 4.3. Figures 7 and 8 show the visualisation of the SONAR data on the 3D model of the void.

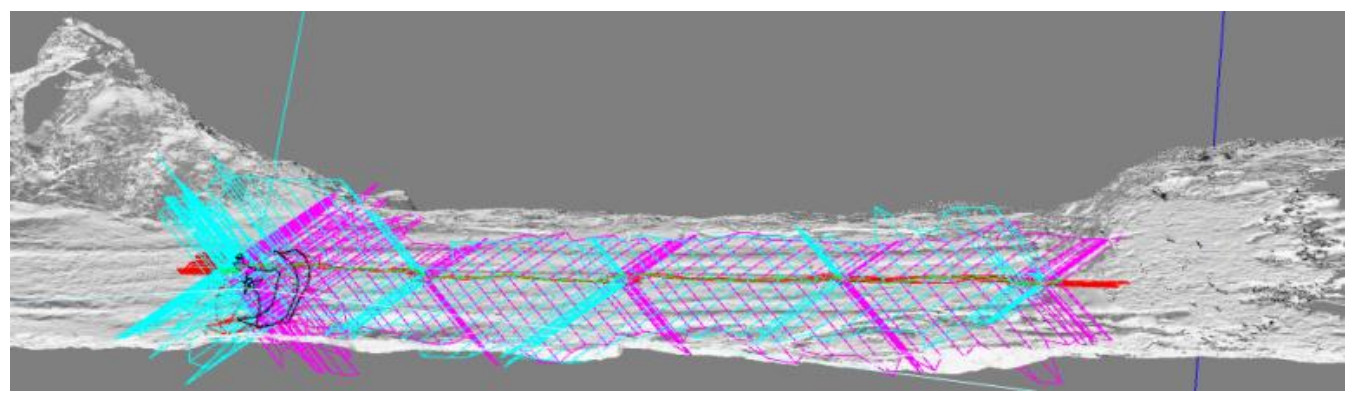

Figure 7 Top view of distances measured by SONAR sensors in Experiment 2; cyan for the front and back-left SONAR sensors and magenta for the front and back-right SONAR sensors

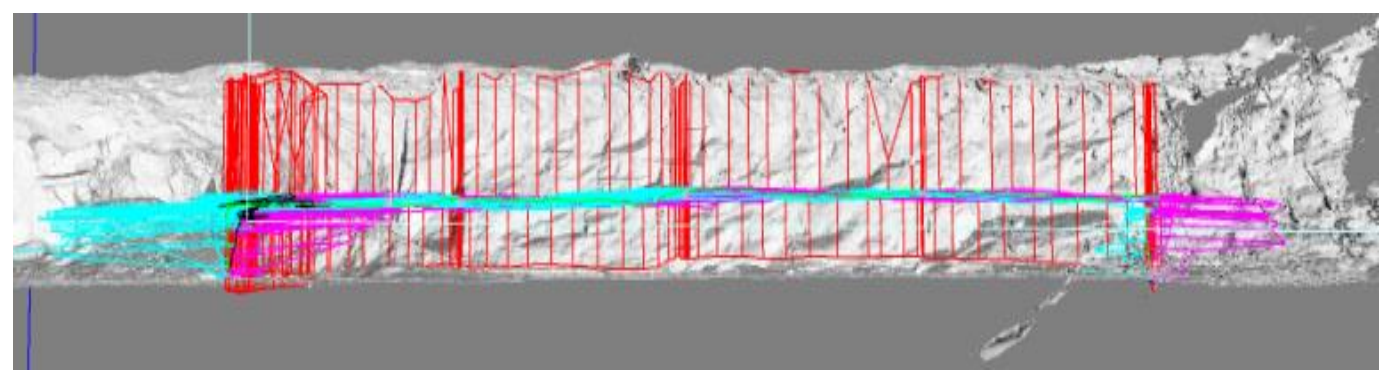

Figure 8 Right side view of distances measured by SONAR sensors in Experiment 2; where red lines indicate the upwards and downwards-facing SONAR

\subsection{Aligning IMU data and SONAR data}

Refer to Subsection 4.4.

\subsection{Constraints}

With the electrical noise filtered out (Section 4.5), the camera was able to maintain its frame rate throughout the whole experiment. However, the positioning algorithm failed to produce a reliable trajectory of the UAV in certain cases. This might be due to the surface not reflecting the light at times, when the wall or floor were too far away from the light source, causing the algorithm to be unable to detect any visual ques from the image produced by the camera on-board, hence no optical flow was able to be deduced from it. 


\section{$6 \quad$ Results}

The accuracy of each SONAR sensor was evaluated by calculating the difference in metres between the distance between corresponding points on the ceiling/wall/floor of the cavern to the sensor and distance given by the sensor data. This means that an error with negative values indicates that the distance detected by the SONAR sensors is larger than the ground truth baseline, in this case the corresponding points on the wall/ceiling/floor of the cavern, and smaller if values are positive. Statistics calculated and presented in the following tables include mean, absolute (abs.) mean - the mean of the absolute values, absolute maximum (max.), absolute minimum (min.) and standard deviation (std). Tables 1 and 2 show the statistics of the accuracy of each SONAR sensor in Experiment 1 and Experiment 2 respectively.

Table 1 Accuracy of each SONAR sensor in Experiment 1, in metres

\begin{tabular}{lllllll}
\hline SONAR position & Front-right & Back-right & Front-left & Back-left & Down & Up \\
\hline Mean & 0.1763106 & 0.230753 & -0.08463 & -0.16502 & 0.117448 & 0.193607 \\
Abs. mean & 0.2335073 & 0.27864 & 0.215475 & 0.213115 & 0.119788 & 0.242937 \\
Abs. max. & 0.6468763 & 0.604239 & 0.574451 & 0.320764 & 0.290179 & 0.698032 \\
Abs. min. & 0.0109032 & 0.020368 & 0.008937 & 0.002521 & 0.001276 & 0.004332 \\
Std. & 0.2461703 & 0.225781 & 0.210313 & 0.160385 & 0.087365 & 0.255161 \\
\hline
\end{tabular}

Table 2 Accuracy of each SONAR sensors in Experiment 2, in metres

\begin{tabular}{lllllll}
\hline SONAR position & Front-right & Back-right & Front-left & Back-left & Down & Up \\
\hline Mean & 0.062297 & 0.116947 & 0.035208 & -0.01451 & -0.02869 & 0.069767 \\
Abs. mean & 0.258363 & 0.294896 & 0.188421 & 0.204647 & 0.206341 & 0.140591 \\
Abs. max. & 0.268477 & 0.217267 & 0.083792 & 0.136425 & 0.225695 & 0.18161 \\
Abs. min. & $3.04 \mathrm{E}-02$ & $7.62 \mathrm{E}-02$ & $2.06 \mathrm{E}-02$ & 0.024424 & 0.0943 & $6.81 \mathrm{E}-02$ \\
Std. & 0.192235 & 0.167308 & 0.05257 & 0.107858 & 0.228754 & 0.126875 \\
\hline
\end{tabular}

A summary of the statistics are presented in Tables 3 and 4, where the 'Mean' column is the mean of the means and 'Abs. mean' column is the mean of the absolute means of all six SONAR sensors. Despite the up-facing SONAR sensor being of a different model to the other five sensors, it is still included in the statistics since the error of the up-facing SONAR shows no significant difference to the others.

Table 3 Summary of accuracy of all six SONAR sensors in Experiment 1

\begin{tabular}{lll}
\hline & Mean & Abs. mean \\
\hline Mean & 0.078078 & 0.217244 \\
Abs. max. & 0.230753 & 0.27864 \\
Abs. min. & 0.084631 & 0.119788 \\
Std. & 0.163357 & 0.053314 \\
\hline
\end{tabular}


Table 4 Summary of accuracy of all six SONAR sensors in Experiment 2

\begin{tabular}{lll}
\hline & Mean & Abs. mean \\
\hline Mean & 0.04017 & 0.215543 \\
Abs. max. & 0.116947 & 0.294896 \\
Abs. min. & 0.014506 & 0.140591 \\
Std. & 0.054803 & 0.054198 \\
\hline
\end{tabular}

Based on Tables 3 and 4, including all six sensors, the absolute average maximum error in Experiment 1 and Experiment 2 is $0.29 \mathrm{~m}$, with an average absolute mean error of $0.22 \mathrm{~m}$.

\section{Discussion}

During Experiments 1 and 2, the data was captured in two different ways; with the UAV flown, and with it being carried. When the UAV was carried, there were occasions where the UAV operator's body and/or limbs interfered with the SONAR sensors. This occurrence became increasingly obvious when the SONAR data was visualised due to sections of significant error. Thus, these sections of error were manually identified and excluded when the results in Section 6 of this paper were calculated.

In Experiment 1, as seen in Table 3, the average 'absolute mean' error of all the SONAR positions was approximately $0.217 \mathrm{~m}$. This excludes all data that was determined to be obstructed by the UAV operator while it was being carried. The average standard deviation was only $0.053 \mathrm{~m}$, showing that $68 \%$ of the SONAR measurements were within the error range of $0.22 \pm 0.053 \mathrm{~m}$. Similar results were found in Experiment 2, as seen in Table 4, where the average 'absolute mean' of the SONAR positions was $0.216 \mathrm{~m}$ with a standard deviation of $0.054 \mathrm{~m}$.

In Experiment 1, the downwards-facing SONAR, labelled 'Down' in Table 1, showed the least amount of 'absolute mean' error at $0.12 \mathrm{~m}$ with a standard deviation of $0.087 \mathrm{~m}$. This was attributable to the ground remaining at a relatively constant and close distance from the sensor compared to all of the other sensors which detected surfaces at greater and more varying distances.

In Experiment 2, the variance of the data is smaller than the variance in Experiment 1, looking at the absolute mean value in relation to standard deviation value, which means having closer values to the ground truth.

During Experiment 1, the light source that was mounted on the UAV to assist the on-board cameras with photogrammetry created electronic noise that resulted in the camera's frame rate to be significantly decreased. Hence, the on-board lights were inoperable in conjunction with the on-board camera, and only ambient light was available to assist the photogrammetry. There did happen to be some light sources pre-installed in the experiment location, but these were often insignificant, causing the photogrammetry to fail. However, in Experiment 2 this issue was resolved by including an electromagnetic interference filter to the power supply, allowing both the camera and lights to be active simultaneously.

After the first experiment, it was discovered that the range on the SONAR sensors used was limited to only $3 \mathrm{~m}$ due to having a time constraint before accessing the experiment location, as well as an undefined type of sensor and its specifications. Thus, it was deemed that the original location of the experiment was unsuitable since it was located in such a large open area. This was resolved in Experiment 2, where the experiment was relocated to a more constricted area, allowing the walls of the underground area to be within SONAR range at all times.

The quantity of loose dust present in the void in Experiment 1 made it difficult to manually fly the UAV, since the propellers caused large amounts of this dust to become airborne, obstructing the vision of the UAV pilot. This caused the data from all of the flown tests to become unusable, since the pilot was unable to fly the UAV along the necessary path in these conditions. With the path of the drone not following the experiment baseline, it became too difficult to align the IMU's positional data with the photogrammetric model. 


\section{Conclusion}

SONAR sensors are suitable to be used on a UAV, and have the advantage of reduced weight in comparison with LIDAR systems. With a maximum error of around $0.3 \mathrm{~m}$, they are suitable for mapping for operational control since the distance errors can be used as a tolerance in navigation to avoid crashes in both cases of flying the UAV in full autonomy or with human remote piloting. SONAR sensors can support effective real-time operational control of the UAV during void mapping; for example, more detailed maps can be generated after a flight using data from on-board laser scanning and/or video data photogrammetry.

Recommendations for future studies are as follows. The range of SONAR sensors must agree with the size of the void where larger voids require correspondingly longer ranges. Since a SONAR sensor only gives a single range rather than multiple ranges like LIDAR, an array of SONARs could be mounted on a UAV to provide more data more quickly for mapping and navigation. However, this must be tested to identify the impacts in relation to power and weight requirements. Since the accuracy of SONAR data depends on the accuracy of the positioning system, a more robust algorithm should be implemented. In the underground case where GPS is denied, an algorithm that fuses all useful information from available sensors should be implemented. UAV positioning, especially in dark and GNSS-denied environments, provide research opportunities since robust solutions to this problem are still incomplete.

\section{Acknowledgement}

A special thanks to MMG staff for their generous support in bringing us underground; always there to assist and help.

\section{References}

Agisoft LLC 2017, Agisoft Photoscan, viewed 22 May 2017, http://www.agisoft.com

Bloesch, M, Omari, S, Hutter, M \& Siegwart, R 2015, 'Robust visual inertial odometry using a direct EKF-based approach', Proceedings of the 2015 IEEE/RSJ International Conference on Intelligent Robots and Systems (IROS), Institute of Electrical and Electronics Engineers, New York, pp. 298-304.

Cree, Inc. 2017, XLamp XHP70, viewed 24 February 2017, http://www.cree.com/LED-Components-and-Modules/Products/XLamp /Arrays-Directional/XLamp-XHP70

Elfes, A 1990, 'Sonar-based real-world mapping and navigation', in IJ Cox \& GT Wilfong (eds), Autonomous Robot Vehicles, Springer Verlag, Berlin, pp. 233-249.

Engel, J, Schöps, T \& Cremers, D 2014, 'LSD-SLAM: Large-scale direct monocular SLAM', in D Fleet, T Pajdla, B Schiele \& T Tuytelaars (eds), Proceedings of the European Conference on Computer Vision 2014, Springer, Cham, pp. 834-849.

Forster, C, Pizzoli, M \& Scaramuzza, D 2014, 'SVO: Fast semi-direct monocular visual odometry', Proceedings of the IEEE International Conference on Robotics and Automation 2014, Institute of Electrical and Electronics Engineers, New York, pp. 15-22.

Hustrulid, WA \& Bullock, RC 2001, Underground Mining Methods: Engineering Fundamentals and International Case Studies, Society for Mining, Metallurgy, and Exploration, Littleton.

InfoMine 2008, GPS for Underground Operations - Great Potential for Controlling Block Caves, Saving Trapped Miners and Machine Automation, viewed 23 February 2017, http://www.mining.com/gps-for-underground-operations-great-potential-forcontrolling-block-caves-saving-trapped-miners-and-machine-automation/

Klein, G \& Murray, D 2007, 'Parallel tracking and mapping for small AR workspaces', Proceedings of the 6th IEEE and ACM International Symposium on Mixed and Augmented Reality, Institute of Electrical and Electronics Engineers, New York, pp. 225-234.

MaxBotix Inc. 2012a, 12CXL-MaxSonar ${ }^{\circledR}$ EZ ${ }^{\text {TM }}$ Series, High Performance Sonar Rangefinder, MB1242, viewed 24 February 2017 http://www.maxbotix.com/documents/I2CXL-MaxSonar-EZ_Datasheet.pdf, p. 11.

MaxBotix Inc. 2012b, I2CXL-MaxSonar ${ }^{\circledR}$-WR/WRC Series, High Performance Sonar Rangefinder, MB7040, viewed 24 February 2017 http://www.maxbotix.com/documents/I2CXL-MaxSonar-WR_Datasheet.pdf, p. 10.

Open Source Robotics Foundation, Inc. 2017, RViz, viewed 22 May 2017, http://wiki.ros.org/rviz

Thrun, S, Burgard, W \& Fox, D 2005, Probabilistic Robotics, The MIT Press, Cambridge. 